\title{
Reduction of Spatially Correlated Speckle in Textured SAR Images
}

\author{
OLEKSII RUBEL, VLADIMIR LUKIN, SERGIY KRIVENKO, VLADIMIR PAVLIKOV, \\ SIMEON ZHYLA, EDUARD TSERNE \\ National Aerospace University, 17, Chkalov St., Kharkiv, 61070, Ukraine, \\ (E-mail: o.rubel@khai.edu,v.lukin@khai.edu, s.kryvevnko@khai.edu, v.pavlikov@khai.edu, s.zhyla@khai.edu, e.tserne@khai.edu)
}

Corresponding author: Oleksii Rubel (e-mail: o.rubel@khai.edu).

\begin{abstract}
: ABSTRACT Synthetic aperture radars (SARs) provide a lot of images that can be used for numerous applications. A problem with acquired images is that they are corrupted by speckle which is a noise-like phenomenon with multiplicative nature. In addition, speckle is non-Gaussian and it is often spatially correlated. A typical task in SAR image processing is despeckling and many methods have been already proposed. However, most of them do not take noise spatial correlation into account during denoising. In this paper, we show how this can be done in despeckling based on discrete cosine transform. The use of frequency-dependent thresholds leads to sufficient improvement of denoising efficiency in terms of visual quality metrics. Moreover, we consider quite complex structure texture images for which noise removal is usually problematic and can lead to information loss. Comparison to the well-known local statistic Lee and Frost filters, extended DCT-based filter is carried out for different remote sensing systems including Sentinel-1 and Sentinel-2.
\end{abstract}

: KEYWORDS Synthetic aperture radar image; Denoising; Despeckling efficiency; Visual quality.

\section{INTRODUCTION}

$\mathrm{R}$ ADAR remote sensing that is currently mostly represented by airborne and spaceborne synthetic aperture radars is widely employed in various applications [1-3]. Modern SARs are able to acquire images during day and night as well as in bad weather conditions with appropriate spatial resolution of several meters. The problem of SAR images is that they suffer from noise-like phenomenon called speckle [4-6]. This problem has been understood for many years started from fundamental works of J.-S. Lee [7] and V. Frost [8]. The necessity of efficient despeckling of SAR images has been mentioned in numerous papers [9-11]. Filters that belong to different groups have been proposed starting from scanning window ones $[7,8$, 12], continued by denoisers based on orthogonal transforms $[13,14]$, and finalized by modern non-local approaches [1517]. Their performance steadily improves but it is still desired to suppress speckle and preserve heterogeneities (details, edges, textures) better. The practical cases for which a limited success has been gained are complex structure images (those ones containing a lot of edges and fine details and/or highly textured ones) corrupted by spatially correlated speckle. The main reasons for this are the following. Firstly, it is hard to reach high efficiency of filtering for complex structure images (see examples in Fig. 1) even potentially [18-20]. Meanwhile, texture features and fine details have to be preserved at image denoising stage [21, 22]. Secondly, it is especially difficult to discriminate texture and noise if noise is spatially correlated since both statistical and spatial characteristics of signal and noise are quite similar. Thirdly, the case of spatially correlated noise is paid much less attention than a simpler case of white noise. In this sense, it is worth mentioning the papers [14, 23-25] specially devoted to considering reduction of spatially correlated noise.

Thus, our primary goal is to analyze despeckling efficiency for highly textured images corrupted by spatially correlated speckle. Note that speckle is spatially correlated for images acquired by such modern SAR systems as TerraSAR-X (see examples in Fig. 1) and Sentinel-1 [13] (see examples in Fig. 2). The novelty of the presented results 
is that we show how spatial correlation of the speckle can be taken into account in despeckling based on discrete cosine transform (DCT) and evaluate the benefits quantitatively with particular attention to highly textured images.

The paper structure is the following. In Section 2, we briefly analyze an image/noise model and its main properties. Section 3 deals with criteria of image despeckling efficiency. Simulation data and their analysis are given in Section 4. Real data example is given as well. Finally, the conclusions are presented.

\section{IMAGE/NOISE MODEL}

A traditional model of SAR images is based on assumption that speckle is pure multiplicative $[4,7-10]$ that has been confirmed by many tests $[4,6]$. Then, the noisy (acquired) image is then described as $I_{i j}^{s p}=I_{i j}^{\text {true }} \mu_{i j}$, i.e. the product of a true value and random multiplicative factor where ij are indices of image pixels. For pure multiplicative noise, it is assumed that speckle has constant (relative) variance equal to $\sigma_{\mu}^{2}=0.273 / L$ where $L$ is the number of looks. For real life data, this parameter can be either determined using a priori information on operation principles and characteristics of a given SAR, or experimentally as $\delta_{\mu_{i j}}^{2}=\sigma_{i j}^{2} / \bar{I}_{i j}^{2} \sigma_{\mu}^{2}$, where $\sigma_{i j}^{2}$ is the local variance estimate determined for a manually or automatically chosen homogeneous image region and $\bar{I}_{i j}^{2}$ is the mean for this fragment. $\sigma_{\mu}^{2}$ varies within the limits from 0 to 1 depending SAR characteristics, for single look amplitude speckle $\sigma_{\mu}^{2}=0.273$ - this is just the case in Fig. 1 where many parts (regions) of the image in Fig. 1(b) can be used for getting the speckle variance estimate.

Fig. 2 gives examples of SAR image fragments acquired by Sentinel-1 for two polarizations: vertical-vertical (vv) and vertical-horizontal (vh). Red color squares show manually chosen fragments for which speckle variance has been estimated and occurred to be slightly larger than 0.05 (i.e. $\mathrm{L}$ is about 5). Spatial correlation of the speckle can be characterized in different manner. It is possible to estimate two-dimensional (2D) autocorrelation function for manually chosen quasi-homogeneous regions of a certain size (e.g., $32 \times 32$ or $64 \times 64$ pixels). However, in our case, it is better to estimate $8 \times 8$ spectrum in discrete cosine transform (DCT) domain. The reasons for this are the following. Firstly, this spectrum is needed in DCT-based denoising adapted to noise properties (see the details in Section 4). Secondly, for such size, spectrum estimates can be averaged well since it is usually quite easy to find quasi-homogeneous image regions totally containing thousands of pixels.

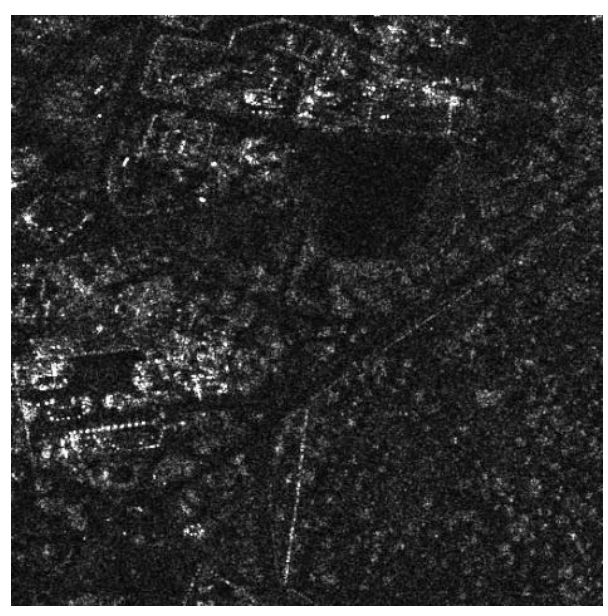

(a)

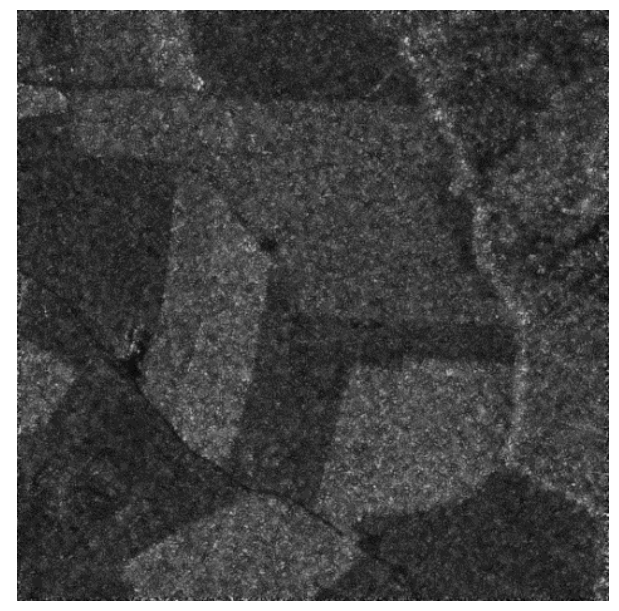

(b)

Figure 1. Single-look SAR image fragments of complex (a) and simple (b) structures

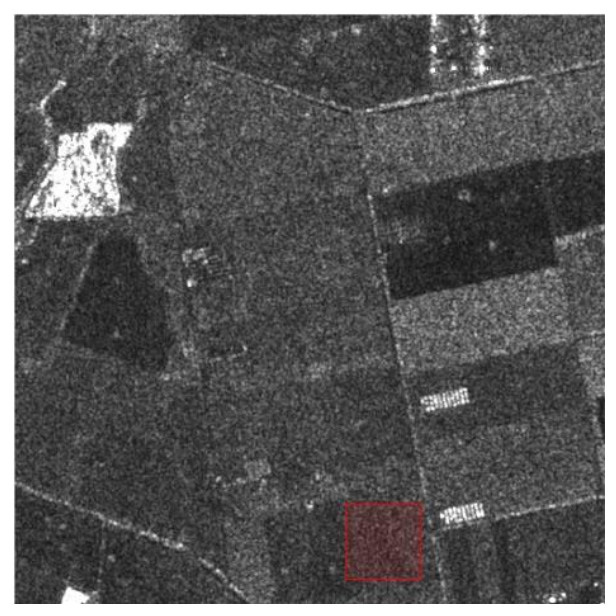

(a) 


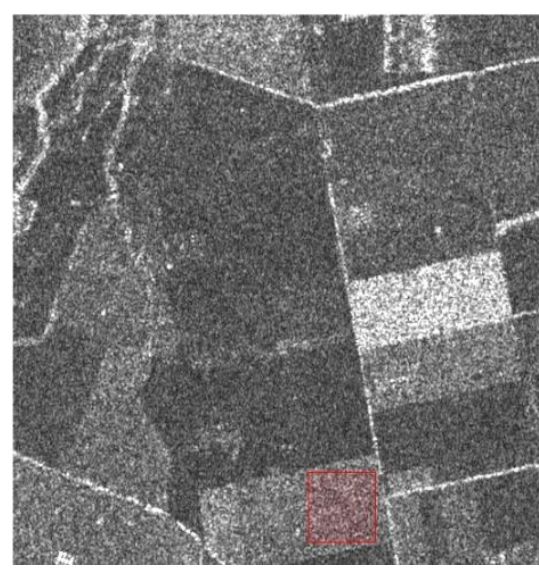

(b)

Figure 2. SAR image fragments of vv (a) and vh (b) polarizations with marked homogeneous regions

Examples of normalized DCT spectrum estimates for image fragments in Fig. 2 are given in Fig. 3. Here some explanations are needed. Small indices correspond to low spatial frequencies. Thus, spectrum is, obviously, non-uniform that evidences in favor of conclusion that speckle in spatially correlated.

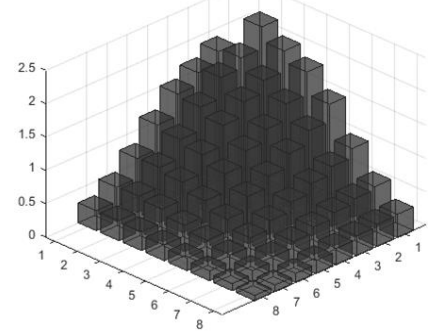

(a)

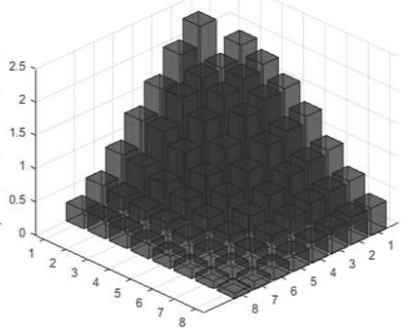

(b)
Figure 3. DCT spectrum estimates for vv (a) and vh (b) polarization images

Then, to get quantitative evaluation of speckle suppression efficiency, we need means for modelling speckle with the determined properties. Methods to do this have been earlier described in our papers [23, 26]. In Fig. 4, we give an example of noise-free and three noisy images with spatially correlated speckle with different properties.

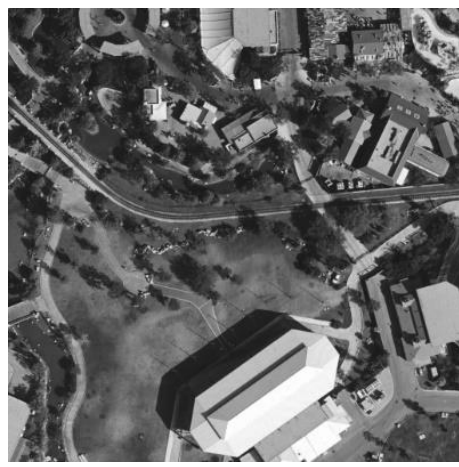

(a)

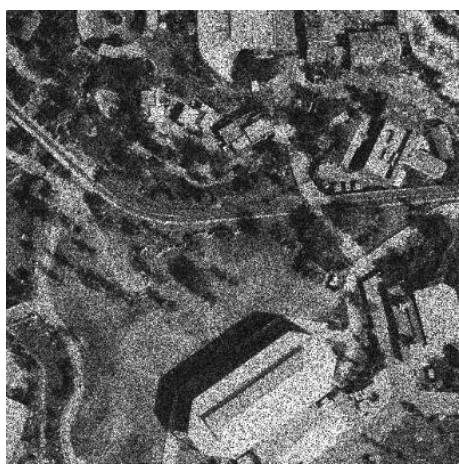

(b)

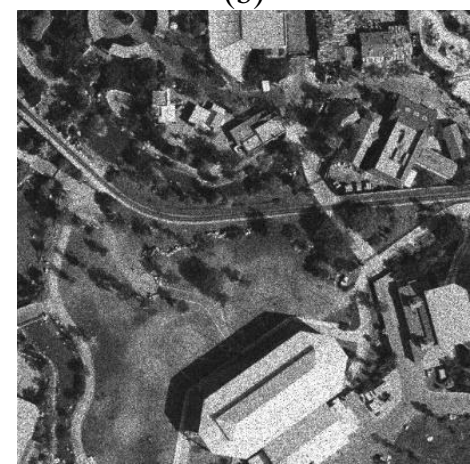

(c)

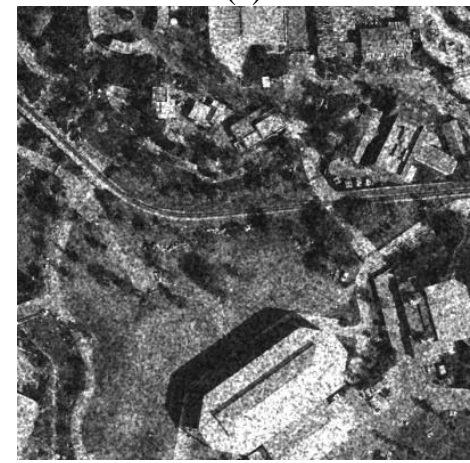

(d)

Figure 4. Noise-free test image (a) and its noisy versions for single-look speckle (b) and five looks with smaller (c) and higher (d) degrees of spatial correlation

Noise-free image, see Fig. 4(a) contains homogeneous regions and many objects of different shapes and contrasts with respect to surrounding background as well as fine details. Image in Fig. 4(b) corresponds to the case of amplitude single-look speckle with low degree of spatial correlation. Obviously, many small-sized objects (especially if they have low contrast) cannot be easily recognized. Fig. 4(c) shows the image with the same spatial correlation properties of speckle but with $L=5$. The image looks more pleasant compared to the image in Fig. 4(b) and more smallsized objects can be reliably detected and localized. Fig. 4(d) presents 5-look image but with speckle with considerably larger degree of spatial correlation than in image in Fig. 4(c). Its appearance (visual quality) is sufficiently worse and it is difficult to judge what image is better, in Fig. 4(b) or 4(d). 
This example shows one more problem with spatially correlated noise in general and speckle in particular. Images corrupted by noise with higher degree of spatial correlation are perceived as those one having worse visual quality. Thus, in addition to traditional criteria of original image quality and efficiency of their denoising, it is worth applying visual quality criteria. Note that it is still a question what are the best visual quality criteria for applicable in SAR image analysis and processing. Obviously, this should be metrics applicable for grayscale images and possessing good adequateness with respect to peculiarities of human vision system (HVS). In this sense, we can further employ earlier experience $[22,26]$ concerning visual quality metrics [27-30] and their use in SAR image processing [26].

Alongside with test images given in Fig. 4, we are more interested in special kinds of textural images. This is because it is known that the task of improving image quality due to denoising is the hardest just for highly textured images $[18,26]$. One way to simulate them is to apply fractals. Another way is to use images acquired by Sentinel-2. Two examples of noise-free images are represented in Fig. 5. Their noisy versions with noise having properties similar to Sentinel-1 images are shown in Fig. 6. Fig. 7 gives an example of the image with many details. Obviously, noise distorts textures and, simultaneously, texture masks noise. The task is then to remove noise with texture feature preservation.

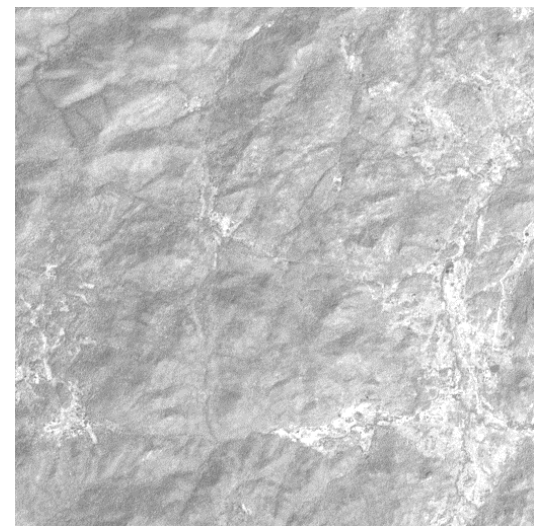

(a)

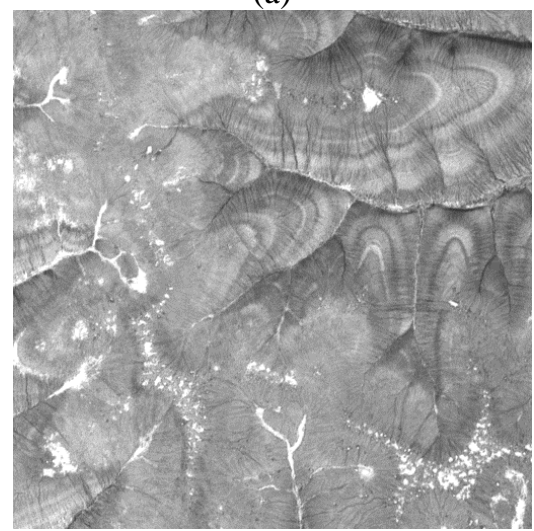

(b)

Figure 5. Examples of noise-free textures

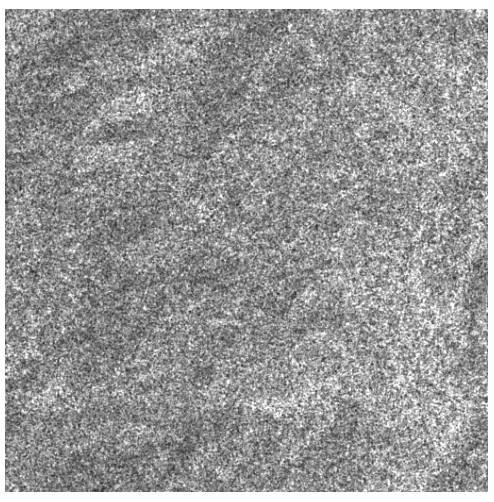

(a)

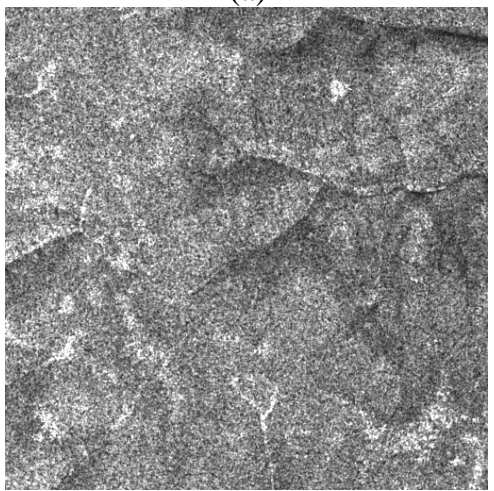

(b)

Figure 6. Noisy versions of images in Fig. 5 with PSNR about $17.5 \mathrm{~dB}$ (a), PSNR-HVS-M is about $14.8 \mathrm{~dB}$ (b)

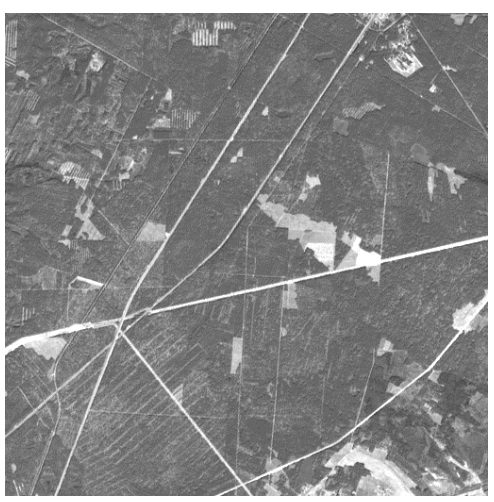

(a)

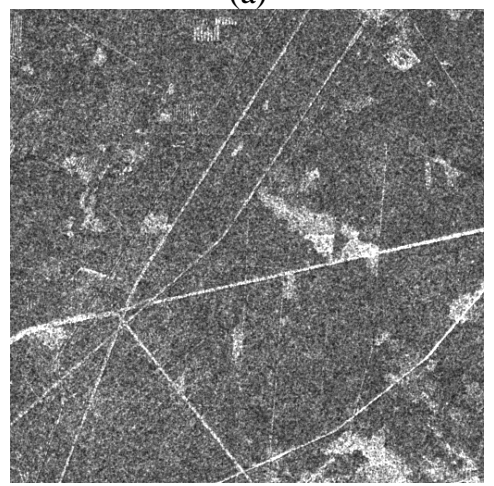

(b)

Figure 7. Noise-free (a) and noisy (b) images with many details, PSNR=19.5 dB, PSNR-HVS-M=16.9 dB 


\section{QUANTITATIVE CRITERIA AND PRINCIPLE OF DCT-} BASED DENOISING

One traditional criterion - peak signal-to-noise ratio (PSNR) - has been already mentioned. In simulations, it can be determined as

$$
\begin{gathered}
P S N R^{i n p}=10 \log _{10}\left(D^{2} / M S E^{i n p}\right), \\
M S E^{i n p}=\sum_{i=1}^{I} \sum_{j=1}^{J}\left(I_{i j}^{\text {noise }}-I_{i j}^{\text {true }}\right)^{2} /(I J),
\end{gathered}
$$

where $I$ and $J$ determine image size, $D$ is image representation dynamic range equal to 255 in the considered case. PSNR is expressed in $\mathrm{dB}$ where a larger value relates to a better quality.

Another used metric is PSNR-HVS-M (http://www.ponomarenko.info/psnrhvsm.htm) based on HVS. The following two important properties of HVS are exploited: a higher sensitivity to distortions in low spatial frequencies and masking effect. These effects are incorporated in calculation of MSE-HVS-M used in calculation of PSNRHVS-M as

$$
\begin{aligned}
& P S N R-H V S-M^{i n p}= \\
& 10 \log _{10}\left(D^{2} / M S E_{H V S-M}^{i n p}\right),
\end{aligned}
$$

Suppose now that we have despeckled images $\left\{I_{i j}^{d}, i=1, \ldots, I, j=1, \ldots, J\right\}$. Then it is possible to calculate

$$
\begin{aligned}
& M S E^{d}=\sum_{i=1}^{I} \sum_{j=1}^{J}\left(I_{i j}^{d}-I_{i j}^{\text {true }}\right)^{2} /(I J), \\
& P S N R^{d}=10 \log _{10}\left(D^{2} / M S E^{d}\right), \\
& P S N R-H V S-M^{d}= \\
& 10 \log _{10}\left(D^{2} / M S E_{H V S-M}{ }^{d}\right),
\end{aligned}
$$

where MSE $_{\mathrm{HVSM}}$ is calculated for filtered and noise-free images. PSNR-HVS-M is also expressed in $\mathrm{dB}$ and its larger values correspond to better visual quality.

Having input and output values of the considered metrics, it is possible to calculate their improvements as:

$$
\delta P S N R=P S N R^{d}-P S N R^{i n p},
$$

$\delta P S N R-H V S-M=$

PSNR-HVS - $M^{d}-P S N R-H V S-M^{i n p}$, where both improvements are in $\mathrm{dB}$ and their larger values, in general, correspond to more efficient denoising.

We have numerous options in choosing filters for efficiency analysis [22]. Even though many filters are not suited for multiplicative noise removal, preliminary homomorphic transform of logarithmic type can be used to convert pure multiplicative noise into pure additive [20] (although not Gaussian). Meanwhile, many filters can not be directly adapted to suppression of spatially correlated noise and this might make worse their performance. Due to this obstacle, we further consider DCT-based filters [13] that can be easily adapted to both multiplicative nature and spatial correlation of speckle.

More in detail, image despeckling is carried out in the following four steps. Firstly, direct 2D DCT is performed for each $8 \times 8$ pixel block. In this way, 64 DCT coefficients are obtained. Secondly, they are thresholded (only the DCTcoefficient relating to the block mean is kept unchanged). Several variants of thresholding are possible. Here we consider hard thresholding. For conventional DCT-filter, for each nm-th block the threshold is set as $T(n, m)=\beta \sqrt{f(\bar{I}(n, m))}$ where $\beta$ denotes the filter parameter commonly set equal to $2.7, \bar{I}(n, m)$ denotes the local mean in the block, $f(\bar{I}(n, m))$ is the function that characterizes dependence of the local variance on local mean; then, for pure multiplicative noise one has $T(n, m)=\beta \sqrt{f(\bar{I}(n, m))}$. For the modified DCT-based filter $[13,26]$ adapted to speckle spatial correlation, one has frequency dependent thresholds defined as $T(n, m, k, l)=\beta \sigma_{\mu} \bar{I}(n, m) \sqrt{W(k, l)} \quad$ where $W(k, l), k=0, \ldots, 7 ; 0, \ldots, 7 \quad$ is the aforementioned normalized power DCT spectrum for $8 \times 8$ pixels. Then, inverse 2D DCT in each block is applied to thresholded DCT at the third step. This leads to obtaining preliminary filtered values for all pixels belonging to a given block. Note that filtering is usually performed for fully overlapping blocks. This means that neighbor block positions differ by only one pixel in horizontal or vertical directions. Then, a given pixel in most cases belongs to 64 blocks and many preliminary denoised values correspond to it. Usually, these values are averaged for each given pixel and a final filtered value is obtained (this is treated as the fourth step). Let us call this version of DCT-based filter as spatial spectrum adapted (SSA) one. In general, the DCT-based denoising with full overlapping is the most efficient option (among possible variants) - it provides minimal output MSE and MSE ${ }_{\text {HVS-M. }}$. Below we consider DCT-based filters with full overlapping of blocks. Note that due to existence of fast algorithms, the filters are very fast. 


\section{ANALYSIS OF EXPERIMENTAL RESULTS}

Therefore, we consider the performance of conventional and SSA versions of the DCT-based filter. For comparison purpose, we have also analyzed data for the following wellknown denoising techniques: the local statistic $5 \times 5$ and $7 \times 7$ Lee filter [7] as well as 5x5 and 7x7 Frost filter [8].

Fig. 8 shows the $5 \times 5$ Lee filter outputs for images given in Fig. 6. Noise is suppressed and there are considerable improvements of PSNR and PSNR-HVS-M. Meanwhile, there is some smearing observed in output images.

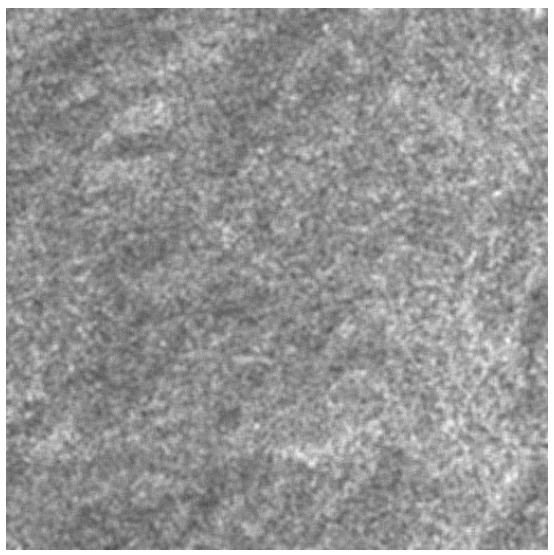

(a)

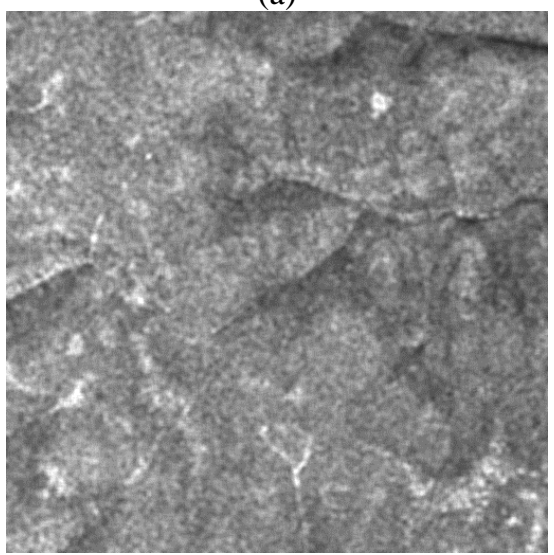

(b)

Figure 8. 5x5 Lee filter outputs for noisy images in Fig. 6: PSNR=24.1 dB, PSNR-HVS-M=19.3 dB (a); PSNR=24.2 $\mathrm{dB}$, PSNR-HVS-M=19.7 dB (b)

Output images for two versions of DCT filters are presented in Figs 9 and 10. Analysis of images in Fig. 9 shows that noise suppression is poor and improvements are less than for the Lee filter. Artifacts are observed. Thus, conventional DCT-based filter is not worth applying. Analysis of output images in Fig. 10 shows that noise suppression is much better and this version outperforms the local statistic Lee filter both in terms of PSNR and PSNRHVS-M. However, there are specific artifacts in the form of small vertical or horizontal "sticks" that are not "detected" by the quality criteria used but which are undesired. This shows that, in additional to quantitative criteria (metrics) analysis, visual inspection is worth applying.

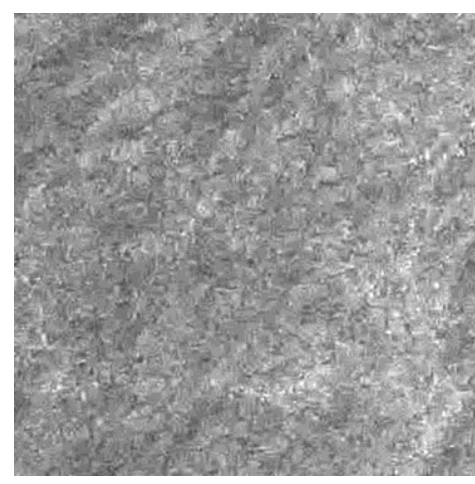

(a)

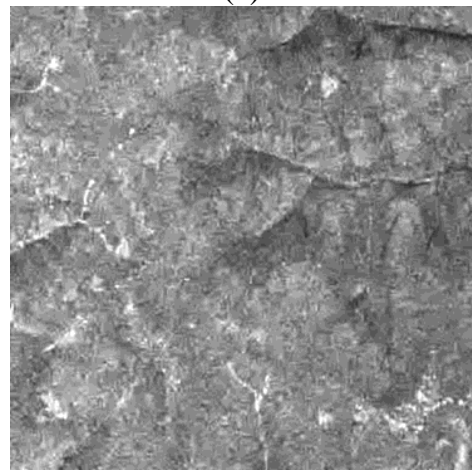

(b)

Figure 9. Conventional DCT-based filter outputs for noise images in Fig. 6: PSNR=23.3 dB, PSNR-HVS-M=18.7 dB (a); PSNR=23.3 dB, PSNR-HVS-M=18.8 dB (b)

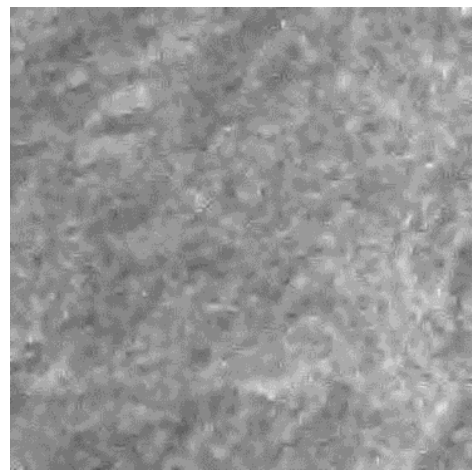

(a)

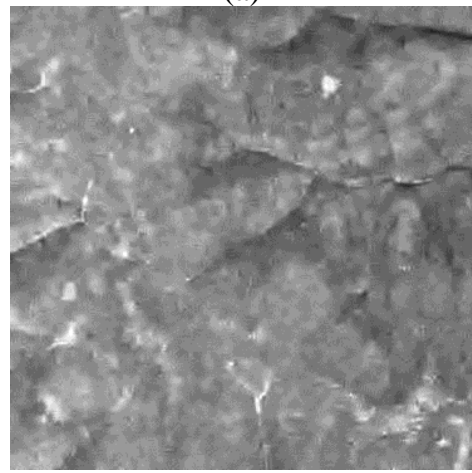

(b)

Figure 10. SSA DCT-based filter outputs for noise images in Fig. 6: PSNR=26.7 dB, PSNR-HVS-M=22.5 dB (a); $\mathrm{PSNR}=25.9 \mathrm{~dB}, \mathrm{PSNR}-\mathrm{HVS}-\mathrm{M}=21.8 \mathrm{~dB}(\mathrm{~b})$ 
Table 1 gives simulation results for despeckling the test image in Fig. 6(a) for all considered filters. Output values of PSNR, PSNR-HVS-M and FSIM [27] are given (the larger the better). In turn, Table 2 presents data for the test image in Fig. 6(b).

As one can see, all the considered filters improve the image quality according to the used quality metrics and this improvement is sufficient. Meanwhile, the results for the modified DCT filter are the best or very close to the best.

Table 1. Comparison data for despeckling the image in Fig. 6(a); input FSIM is equal to 0.611

\begin{tabular}{|c|c|c|c|}
\hline Filter type & $\begin{array}{c}\text { Output } \\
\text { PSNR }\end{array}$ & $\begin{array}{c}\text { Output PSNR- } \\
\text { HVS-M }\end{array}$ & Output FSIM \\
\hline $5 \times 5$ Lee & 24.1 & 19.3 & 0.776 \\
\hline $7 \times 7$ Lee & 25.5 & 21.0 & 0.828 \\
\hline $5 \times 5$ Frost & 23.8 & 18.9 & 0.769 \\
\hline $7 \times 7$ Frost & 25.1 & 20.4 & 0.820 \\
\hline DCT & 23.3 & 18.6 & 0.755 \\
\hline SSA DCT & 26.7 & 22.5 & 0.827 \\
\hline
\end{tabular}

Table 2. Comparison data for despeckling the image in Fig. 6(b); input FSIM is equal to 0.692

\begin{tabular}{|c|c|c|c|}
\hline Filter type & $\begin{array}{c}\text { Output } \\
\text { PSNR }\end{array}$ & $\begin{array}{c}\text { Output PSNR- } \\
\text { HVS-M }\end{array}$ & Output FSIM \\
\hline $5 \times 5$ Lee & 24.2 & 19.7 & 0.819 \\
\hline $7 \times 7$ Lee & 25.0 & 20.7 & 0.839 \\
\hline $5 \times 5$ Frost & 23.9 & 19.3 & 0.814 \\
\hline $7 \times 7$ Frost & 24.7 & 20.2 & 0.836 \\
\hline DCT & 23.4 & 18.9 & 0.800 \\
\hline SSA DCT & 25.9 & 21.8 & 0.835 \\
\hline
\end{tabular}

Consider now the filter outputs for the test image with more details (Fig. 7). The image denoised by the $5 \times 5$ Lee filter is depicted in Fig. 11. PSNR improvement is almost 4 $\mathrm{dB}$, improvement of PSNR-HVS-M is about $3 \mathrm{~dB}$. The outputs for the two versions of DCT-based filter are given in Fig. 12. Again, the conventional version (Fig. 12(a) does not perform well since criteria improvements are quite small. The SSA DCT-based filter output is presented in Fig. 12(b). Improvements of metrics' values are considerable (they are sufficiently greater than improvements for the $5 \times 5$ Lee filter). Noise suppression is clearly visible (compare to images in Fig. 7). However, artifacts are again visible as well.

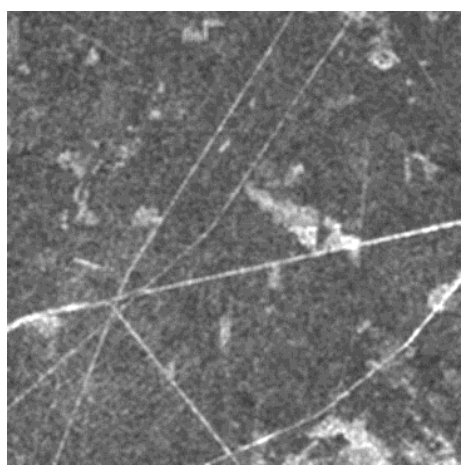

Figure 11. Lee filter outputs for noise image in Fig. 7(b): PSNR=23.4 dB, PSNR-HVS-M=19.9 dB

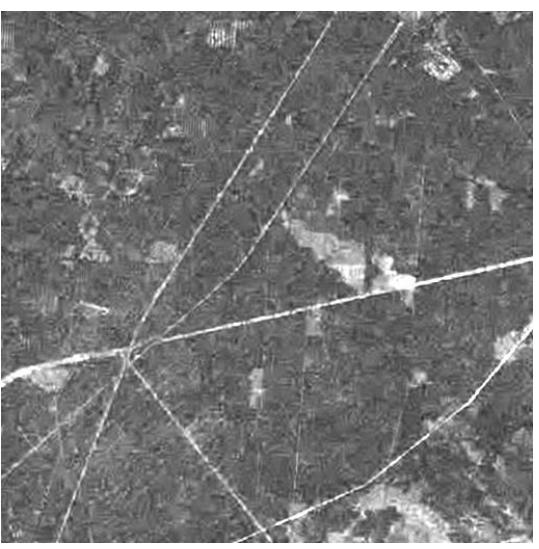

(a)

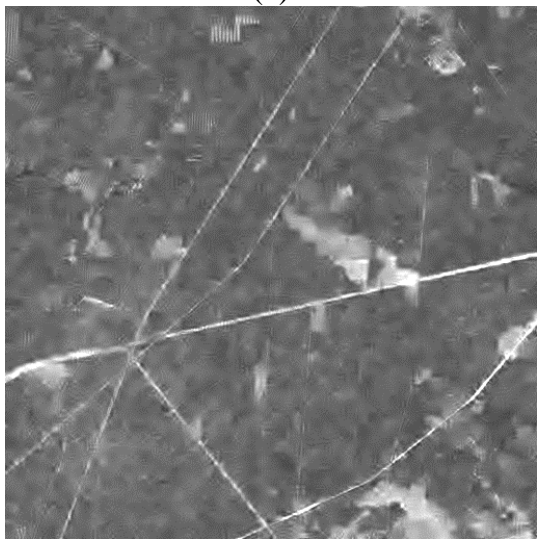

(b)

Figure 12. DCT-based filters' outputs for noise images in

Fig. 7: PSNR=23.8 dB, PSNR-HVS-M=19.9 dB (a);

PSNR $=25.0 \mathrm{~dB}$, PSNR-HVS-M=21.4 dB (b)

Let us give the obtained quantitative data (Table 3). Here we can observe the case when $5 \times 5$ Lee, $5 \times 5$ Frost, and standard DCT filters produce practically the same quality outputs. Meanwhile, the modified version adapted to speckle spatial correlation clearly outperforms all aforementioned filters.

Table 3. Comparison data for despeckling the image in Fig. 7(b); input FSIM is equal to 0.761

\begin{tabular}{|c|c|c|c|}
\hline Filter type & $\begin{array}{c}\text { Output } \\
\text { PSNR }\end{array}$ & $\begin{array}{c}\text { Output PSNR- } \\
\text { HVS-M }\end{array}$ & Output FSIM \\
\hline $5 \times 5$ Lee & 23.5 & 20.0 & 0.831 \\
\hline $7 \times 7$ Lee & 23.2 & 19.5 & 0.806 \\
\hline $5 \times 5$ Frost & 23.5 & 19.9 & 0.831 \\
\hline $7 \times 7$ Frost & 23.4 & 19.7 & 0.818 \\
\hline DCT & 23,7 & 19.9 & 0.831 \\
\hline SSA DCT & 25.9 & 21.5 & 0.840 \\
\hline
\end{tabular}

Finally, Fig. 13 presents a fragments of real life example. VV-polarization Sentinel-1 image with five looks is given in Fig. 13(a). The SSA DCT-filter output is shown in Fig. 13(b). As one can see, speckle is suppressed well whilst textures, edges, and small-sized objects are preserved. 


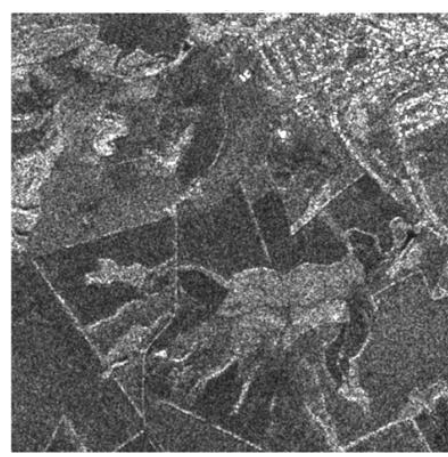

(a)

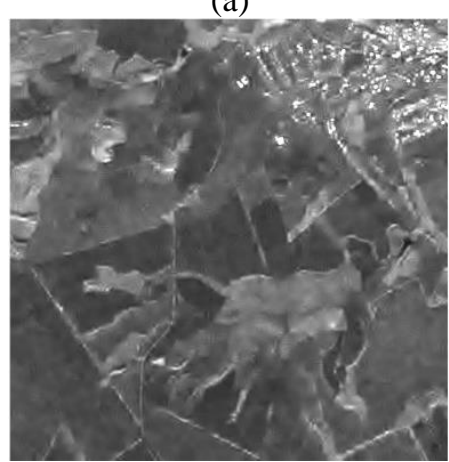

(b)

Figure 13. Original (noisy) SAR image (a) and the SSA DCT filter output (b)

The presented examples show the following:

1) The DCT-based filter is able to preserve textures quite well (in particular, sufficiently better than the local statistic Lee filter) but only if it is adapted to spatial spectrum of spatially correlated noise;

2) In analysis of filter performance, visual inspection is needed since visual quality metrics can evidence in favor of some filters without taking into account the fact that some undesired artifacts are observed in output images; the DCTbased filter adapted to speckle properties produces artifacts that are undesired and can be treated (detected) as low contrast small-sized objects at further stages of SAR image processing.

3) We expect that modifications of nonlocal filters adapted to speckle properties $[15,23]$ can improve despeckling performance according to the metrics used (both traditional and visual quality ones) and the artifacts will be either absent or less seen; probably, not only processing in blocks, but also block search algorithm should be adapted to spatial correlation of the speckle.

\section{CONCLUSIONS}

The task of removal of spatially correlated speckle in textured images is considered. It is demonstrated that, in general, such noise can be suppressed with improvement of PSNR by up to 5-8 dB. Visual quality improves as well. Meanwhile, the sufficient positive effect can be reached if speckle spectral properties are taken into account. This can be done in DCT-based filtering by setting frequency dependent (and, simultaneously, intensity dependent) thresholds. At the same time, if one neglects speckle spatial correlation, this can result in considerable difference between attained and potential efficiencies of denoising. Besides, one has to be careful with artifacts since they can be present even if quantitative criteria show high efficiency of filtering.

\section{References}

[1] J.-S.Lee, E. Pottier, Polarimetric Radar Imaging: From Basics to Applications, CRC Press, 2009, 422 p.

[2] N. Kussul, S. Skakun, A. Shelestov, O. Kussul, "The use of satellite SAR imagery to crop classification in Ukraine within JECAM project," Proceedings of the IEEE International Geoscience and Remote Sensing Symposium (IGARSS), Quebec City, Canada, July 2014 , pp. 1497-1500 https://doi.org/10.1109/IGARSS.2014.6946721.

[3] A. Roth, U. Marschalk, K. Winkler, B. Schättler, M. Huber, I. Georg, C. Künzer, S. Dech, "Ten years of experience with scientific TerraSAR-X data utilization," Remote Sensing, vol. 10, issue 8, pp. 1170, 2018. https://doi.org/10.3390/rs10081170.

[4] C. Oliver, S. Quegan, Understanding Synthetic Aperture Radar Images, SciTech Publishing, 2004, 486 p.

[5] A. G. Mullissa, C. Persello, V. Tolpekin, "Fully convolutional networks for multi-temporal SAR image classification," Proceedings of the IEEE International Geoscience and Remote Sensing Symposium (IGARSS), Valencia, Spain, July 2018, pp. 6635-6638. https://doi.org/10.1109/IGARSS.2018.8518780.

[6] Y. Makinen, L. Azzari, A. Foi, "Exact transform-domain noisevariance for collaborative filtering of stationary correlated noise," Proceedings on IEEE International Conference on Image Processing (ICIP), 22-25 September 2019, pp. 185-189. https://doi.org/10.1109/ICIP.2019.8802964.

[7] J.S. Lee, "Digital image enhancement and noise filtering by use of local statistics," IEEE Transactions on Pattern Analysis and Machine Intelligence, vol. PAMI-2, issue 2, pp. 165-168, 1980. https://doi.org/10.1109/TPAMI.1980.4766994.

[8] V.S. Frost, J.A. Stiles, K.S. Shanmugan, J.C. Holtzman, “A model for radar images and its application to adaptive digital filtering of multiplicative noise," IEEE Transactions on Pattern Analysis and Machine Intelligence, vol. PAMI-4, issue 2, pp. 157-166, 1982. https://doi.org/10.1109/TPAMI.1982.4767223.

[9] R. A. Touzi, "Review of speckle filtering in the context of estimation theory," IEEE Transactions on Geoscience and Remote Sensing, vol. 40, issue 11, pp. 2392-2404, 2002. https://doi.org/10.1109/TGRS.2002.803727.

[10] P. Kupidura, "Comparison of filters dedicated to speckle suppression in SAR images," ISPRS-International Archives of the Photogrammetry, Remote Sensing and Spatial Information Sciences, Prague, Czech Republic, July 12-19, 2016, pp. 269-276. https://doi.org/10.5194/isprsarchives-XLI-B7-269-2016.

[11] C.A. Deledalle, L. Denis, S. Tabti, F. Tupin, "MuLoG, or how to apply Gaussian denoisers to multi-channel SAR speckle reduction?," IEEE Transactions on Image Processing, vol. 26, issue 9, pp. 4389-4403, 2017. https://doi.org/10.1109/TIP.2017.2713946.

[12] J.-S. Lee, J.-H. Wen, T.L. Ainsworth, K-S.Chen, A.J. Chen, "Improved sigma filter for speckle filtering of SAR imagery," IEEE Transactions on Geoscience and Remote Sensing, vol. 47, issue 1, pp. 202-213, 2009. https://doi.org/10.1109/TGRS.2008.2002881.

[13] S. Abramov, O. Rubel, V. Lukin, A. Shelestov, M. Lavreniuk, "Speckle reducing for Sentinel-1 SAR data," Proceedings of the International Geoscience and Remote Sensing Symposium (IGARSS), Fort Worth, TX, USA, December 4, 2017, pp. 2353-2356. https://doi.org/10.1109/IGARSS.2017.8127463.

[14] S. Solbo, T. Eltoft, "A stationary wavelet domain Wiener filter for correlated speckle," IEEE Transactions on Geoscience and Remote Sensing, vol. 46, issue 4, pp. 1219-1230, 2008. https://doi.org/10.1109/TGRS.2007.912718.

[15] M. Matrecano, G. Poggi, L. Verdoliva, "Improved BM3D for correlated noise removal," Proceedings of International Conference on Computer Vision, Theory and Applications, Springer, Rome, 2012, pp. $129-134$

[16] S. Parrilli, M. Poderico, C.V. Angelino, L. Verdoliva, "A nonlocal SAR image denoising algorithm based on LLMMSE wavelet 
shrinkage," IEEE Transactions on Geoscience and Remote Sensing, vol. 50, issue 2, pp. 606-616, 2012. https://doi.org/10.1109/TGRS.2011.2161586.

[17] D. Cozzolino, L. Verdoliva, G. Scarpa, G. Poggi, "Nonlocal CNN SAR image despeckling," Remote Sensing, vol. 12, issue 6, p.1006, 2020. https://doi.org/10.3390/rs12061006.

[18] P. Chatterjee, P. Milanfar, "Is denoising dead?," IEEE Transactions on Image Processing, vol. 19, issue 4, pp. 895-911, 2010. https://doi.org/10.1109/TIP.2009.2037087.

[19] P. Chatterjee, P. Milanfar, "Practical bounds on image denoising: From estimation to information," IEEE Transactions on Image Processing, vol. 20, issue 5, pp. 1221-1233, 2011. https://doi.org/10.1109/TIP.2010.2092440.

[20] P. Milanfar, "A tour of modern image filtering," IEEE Signal Processing Magazine, vol. 30, pp. 106-128, 2013. https://doi.org/10.1109/MSP.2011.2179329.

[21] B. Aiazzi, L. Alparone, S. Baronti, R. Carla, "Adaptive texturepreserving filtering of multitemporal ERS-1 SAR images," Proceedings of the IEEE International Geoscience and Remote Sensing Symposium, Singapore, Singapore, August 3-8, 1997, pp. 2066-2068.

[22] X. Liu, S. Bourennane, C. Fossati, "Nonwhite noise reduction in hyperspectral images," IEEE Geoscience and Remote Sensing Letters, vol. 9 9, pp. $368-372, \quad 2012$. https://doi.org/10.1109/LGRS.2011.2169041.

[23] O. Rubel, V. Lukin, K. Egiazarian, "Additive spatially correlated noise suppression by robust block matching and adaptive 3D filtering," Journal of Imaging Science and Technology, vol. 62, issue 6, pp. 60401-1-60401-11,

2018 https://doi.org/10.2352/J.ImagingSci.Technol.2018.62.6.060401.

[24] B. Goossens, A. Pizurica, W. Philips, "Removal of correlated noise by modeling the signal of interest in the wavelet domain," IEEE Transactions on Image Processing, vol. 18, issue 6, pp. 1153-1165, 2009. https://doi.org/10.1109/TIP.2009.2017169.

[25] M. Lebrun, M. Colom, J. M. Morel, "The noise clinic: A universal blind denoising algorithm," Proceedings of the IEEE International Conference on Image Processing (ICIP), Paris, France, October 2730, 2014, pp. 2674-2678. https://doi.org/10.1109/ICIP.2014.7025541.

[26] O. Rubel, V. Lukin, A. Rubel, K. Egiazarian, "NN-based prediction of Sentinel-1 SAR image filtering efficiency," Geosciences, vol. 9, no. 7, p. 290, 2019. https://doi.org/10.3390/geosciences 9070290 .

[27] L. Zhang, X. Mou, D. Zhang, "FSIM: a feature similarity index for image quality assessment," IEEE Transactions on Image Processing, vol. 20, pp. 2378-2386, 2011. https://doi.org/10.1109/TIP.2011.2109730.

[28] E. Larson, D. Chandler, "Most apparent distortion: Full-reference image quality assessment and the role of strategy," Journal of Electronic Imaging, vol. 19, issue 1, pp. 011006:1-011006:21, 2010. https://doi.org/10.1117/1.3267105.

[29] W. Xue, L. Zhang, X. Mou, A. Bovik, "Gradient magnitude similarity deviation: A highly efficient perceptual image quality index," IEEE Transactions in Image Processing, vol. 23, pp. 684-695, 2014. https://doi.org/10.1109/TIP.2013.2293423.

[30] Z. Wang, E. Simoncelli, A. Bovik, "Multiscale structural similarity for image quality assessment," Proceedings of the Thirty-Seventh Asilomar Conference on Signals, Systems \& Computers, Pacific Grove, CA, USA, 9-12 November 2003, pp. 1398-1402.

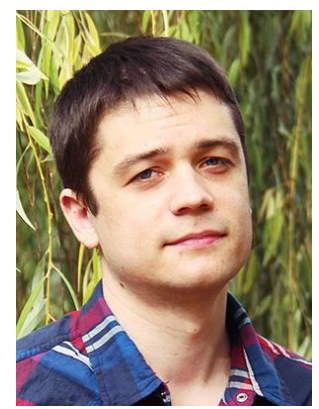

OLEKSII RUBEL received his Ph.D. in Remote Sensing from the National Aerospace University, Ukraine in 2016. Now he is an Associate Professor and pursuing the Doctor in Science degree. His interests include image enhancement and design of image processing algorithms using machine learning.

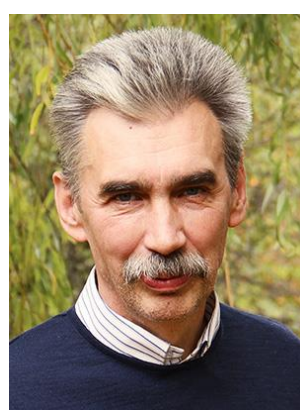

VLADIMIR LUKIN received his Ph.D. in 1988 and Doctor of Technical Science in remote sensing from the National Aerospace University named after M. E. Zhukovsky, Ukraine, in 2002. Currently he is a head of the Department of Information and Communication Technologies and a professor. His research interests include digital signal/image processing, remote sensing data processing, image denoising and compression.

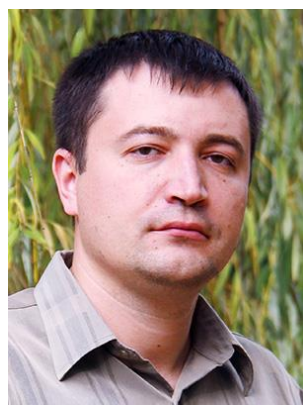

SERGIY KRIVENKO is a senior researcher with PhD in Remote Sensing and 15+ years of research\&industrial experience. He has over fifteen years of successful research experience in digital signal processing $(1 D$ and $2 D$ signal processing including biomedical and physiological signal processing, filtering, compressing and visual quality metrics) and data science.

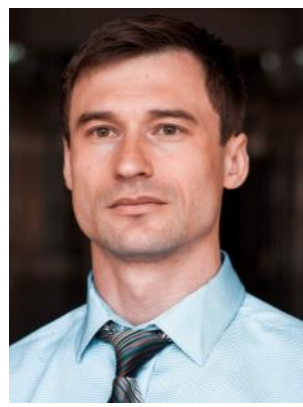

VLADIMIR PAVLIKOV has Doctor of Technical Sciences degree in Radioelectronics. Currently he is a professor of the Department of Aircraft Radioelectronic Systems Design and a vice rector for science in the National Aerospace University. His interests includes spatial-temporal processing of wind-band signals and radar images processing.

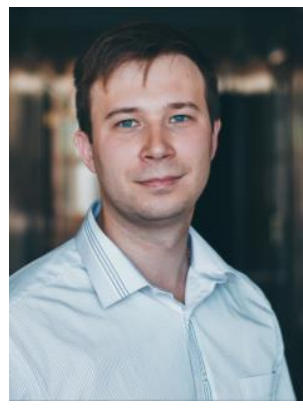

SIMEON ZHYLA has a Candidate of Sciences degree in Radio-electronics. He is a head of the Department of Aircraft Radio-electronic Systems Design in the National Aerospace University. His researches are focused on radar signal reception and processing, aircraft radioelectronic systems designing.

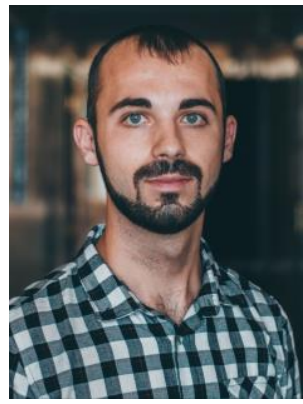

EDUARD TSERNE is a young researcher and PhD student of the Department of Aircraft Radio-electronic Systems Design in the National Aerospace University. He is working on his Ph.D. thesis devoted to radar signal processing. 